Pacific

Journal of

Mathematics

\title{
MARGINALLY TRAPPED SUBMANIFOLDS \\ IN SPACE FORMS WITH ARBITRARY SIGNATURE
}

HENRI ANCIAUX

Volume $272 \quad$ No. 2

December 2014 


\title{
MARGINALLY TRAPPED SUBMANIFOLDS IN SPACE FORMS WITH ARBITRARY SIGNATURE
}

\author{
HENRI ANCIAUX
}

Dedicated to the memory of Franki Dillen (1963-2013).

\begin{abstract}
We give explicit representation formulas for marginally trapped submanifolds of codimension two in pseudo-Riemannian spaces with arbitrary signature and constant sectional curvature.
\end{abstract}

\section{Introduction}

Let $(\mathcal{N}, g)$ be a pseudo-Riemannian manifold and $\mathscr{Y}$ a submanifold of $(\mathcal{N}, g)$ with nondegenerate induced metric. We shall say that $\mathscr{Y}$ is marginally trapped if its mean curvature vector is null, that is, $g(\vec{H}, \vec{H})$ vanishes. When $(\mathcal{N}, g)$ is a Lorentzian fourmanifold and $\mathscr{S}$ is spacelike, the marginally trapped condition has an interpretation in terms of general relativity: it describes the horizon of a black hole [Penrose 1965; Chruściel et al. 2010]. The equation $g(\vec{H}, \vec{H})=0$ is nevertheless interesting in whole generality from the geometric viewpoint, being actually the simplest curvature equation which is purely pseudo-Riemannian: in the Riemannian case this equation implies minimality.

In [Anciaux and Godoy 2012], marginally trapped submanifolds with codimension two have been locally characterized in several simple Lorentzian spaces: the Minkowski space $\mathbb{R}^{n+2}$, the Lorentzian space forms $d \mathbb{S}^{n+2}$ and $\mathrm{Ad} \mathbb{S}^{n+2}$, and the Lorentzian products $\mathbb{S}^{n+1} \times \mathbb{R}$ and $\mathbb{M}^{n+1} \times \mathbb{R}$. Little has been done about marginally trapped surfaces in the case of a manifold with a non Lorentzian metric. In [Chen 2009], flat marginally trapped surfaces of $\mathbb{R}^{4}$ endowed with the neutral metric $d x_{1}^{2}+d x_{2}^{2}-d x_{3}^{2}-d x_{4}^{2}$ have been studied, while Lagrangian marginally trapped surfaces of complex space forms of complex dimension two were characterized in [Chen and Dillen 2007]. Recently marginally trapped surfaces of certain spaces of oriented geodesics have been investigated [Georgiou and Guilfoyle 2014].

The purpose of the present paper is to extend the results of [Anciaux and Godoy 2012] to the case of codimension-two submanifolds in constant curvature spaces

Supported by CNPq (PQ 306154/2011-0) and Fapesp (2011/21362-2).

MSC2010: 53A10, 53C42.

Keywords: marginally trapped submanifolds, pseudo-Riemannian manifolds, mean curvature vector. 
with arbitrary signature, that is, (i) in the pseudo-Euclidean space $\mathbb{R}_{p+1}^{n+2}$ equipped with the inner product of signature $(p+1, q+1)$, and (ii) in the space form $\mathbb{S}_{p+1}^{n+2}$ of signature $(p+1, q+1)$ and sectional curvature 1 (see next section for more precise definition and notation). As in [Anciaux and Godoy 2012], we rely on the use of the contact structure of the set of null geodesics of the ambient space. The congruence of null lines which are normal to a submanifold of codimension two is a Legendrian submanifold with respect to this contact structure. Conversely, given a null line congruence $\mathscr{L}$ which is Legendrian, there exists an infinite-dimensional family of submanifolds, parametrized by the set of real maps $\tau \in C^{2}(\mathscr{L})$, such that the congruence is normal to them. In order to obtain our characterization results, we prove that, given a Legendrian, null line congruence $\mathscr{L}$, the submanifold parametrized by $\tau$ is marginally trapped if and only if the real map $\tau \in C^{2}(\mathscr{L})$ is a root of certain polynomial map with coefficients valued in $C^{2}(\mathscr{L})$.

The paper is organized as follows: Section 1 introduces some notation and gives the precise statements of the results; Section 2 gives a characterization of those submanifolds whose second fundamental tensor is null (Theorem 1), while Section 3 provides a local representation formula which is similar to that of [Anciaux and Godoy 2012] (Theorem 2). In Section 4, an alternative, more global representation formula is given, under certain maximal rank assumption (Theorems 3 and 4). Finally Section 5 attempts to shed light on the ideas in this paper by providing an interpretation of the general construction in terms of contact geometry and explains also the relation between Theorems 2 and 3 in the Lorentzian case.

\section{Statement of results}

We fix throughout three integers $p, q$ and $n$ such that $p+q=n \geq 1$. We shall denote by $\mathbb{R}_{p+1}^{n+2}$ the $(n+2)$-dimensional real vector space equipped with the inner product of signature $(p+1, q+1)$ given by

$$
\langle\cdot, \cdot\rangle=\sum_{i=1}^{p+1} d x_{i}^{2}-\sum_{i=p+2}^{n+2} d x_{i}^{2} .
$$

A nonvanishing vector $v$ of $\mathbb{R}_{p+1}^{n+2}$ is said to be null if $\langle v, v\rangle=0$. We furthermore introduce the hyperquadric

$$
\mathbb{S}_{p+1}^{n+2}:=\left\{x \in \mathbb{R}_{p+2}^{n+3}\langle x, x\rangle=1\right\} .
$$

The induced metric of $\mathbb{S}_{p+1}^{n+2}$, still denoted by $\langle\cdot, \cdot\rangle$, has signature $(p+1, q+1)$ and constant sectional curvature 1. Conversely it is well known (see [Kriele 1999]) that a simply connected $(n+2)$-dimensional manifold endowed with a pseudoRiemannian metric with signature $(p+1, q+1)$ and constant sectional curvature 
is, up to isometries and scaling, $\mathbb{R}_{p+1}^{n+2}$ or $\mathbb{S}_{p+1}^{n+2}$. We shall call these spaces pseudoRiemannian space forms.

We shall be concerned with submanifolds $\Sigma$ of $\mathbb{R}_{p+1}^{n+2}$ and $\mathbb{S}_{p+1}^{n+2}$ with nondegenerate induced metric $g$ and whose normal bundle $N \Sigma$ (i) is two-dimensional (so that $\Sigma$ has dimension $n$ ), and (ii) has indefinite (Lorentzian) metric (so that the induced metric on $\Sigma$ has signature $(p, q))$. We recall that the second fundamental form $h$ of $\Sigma$ is the symmetric tensor $h: T \Sigma \times T \Sigma \rightarrow N \Sigma$ defined by $h(X, Y):=\left(D_{X} Y\right)^{\perp}$, where $(\cdot)^{\perp}$ denotes the projection onto the normal space $N \Sigma$ and $D$ is the LeviCivita connection of ambient space. If $v$ is a normal vector field along $\Sigma$, we define the shape operator of $\Sigma$ with respect to $v$ to be the endomorphism of $T \Sigma$ defined by $A_{v} X=-\left(D_{X} v\right)^{\top}$, where $(\cdot)^{\top}$ denotes the projection onto $T \Sigma$. The relation $\langle h(X, Y), v\rangle=\left\langle A_{v} X, Y\right\rangle$ shows that the second fundamental form and the shape operator carry the same information.

The mean curvature vector $\vec{H}$ of the immersion is the trace of $h$ with respect to the induced metric of $\Sigma$ divided by $n$. Our first result is the characterization of $n$-dimensional submanifolds of space forms with null second fundamental form, that is, such that $h(X, Y)$ is null for all $X, Y \in T \Sigma$ :

Theorem 1. Let $v$ be a constant, null vector of $\mathbb{R}_{p+1}^{n+2}$ and $\Sigma$ an n-dimensional submanifold with nondegenerate induced metric which is contained in the hyperplane $v^{\perp}$. Then $\Sigma$ has null second fundamental form and is therefore marginally trapped. Moreover both the tangent and the normal bundles of $\Sigma$ are flat.

Analogously let $v$ be a constant, null vector of $\mathbb{R}_{p+2}^{n+3}$ and $\Sigma$ an $n$-dimensional submanifold of $\mathbb{S}_{p+1}^{n+2}$ with nondegenerate induced metric which is contained in the hypersurface $v^{\perp} \cap \mathbb{S}_{p+1}^{n+2}$. Then $\Sigma$ has null second fundamental form and is therefore marginally trapped. Moreover $\Sigma$ has constant scalar curvature and flat normal bundle.

Conversely any submanifold of $\mathbb{R}_{p+1}^{n+2}$ or $\mathbb{S}_{p+1}^{n+2}$ with null second fundamental form is locally described in this way.

Quite surprisingly, the method introduced in [Anciaux and Godoy 2012] in the Lorentzian case can be used here, in the case of marginally trapped submanifolds whose second fundamental form is not null, providing local parametrizations:

Theorem 2. Let $\sigma$ be an immersion of class $C^{4}$ of an $n$-dimensional manifold $M$ into $\mathbb{R}_{p+1}^{n+1}$ (respectively, $\mathbb{S}_{p+1}^{n+1}$ ) whose induced metric is nondegenerate and has signature $(p, q)$. Denote by $v$ the Gauss map of $\sigma$, which is therefore $\mathbb{S}_{p}^{n}$-valued (respectively, $\mathbb{S}_{p+1}^{n+1}$-valued), by $A=-d v$ the corresponding shape operator, and by $\tau_{i}$ the roots of the polynomial of degree $n-1$

$$
P(\tau):=\operatorname{tr}(\operatorname{Id}-\tau A)^{-1} .
$$

Then the immersions $\varphi_{i}: \mathcal{M} \rightarrow \mathbb{R}_{p+1}^{n+2}=\mathbb{R}_{p+1}^{n+1} \times \mathbb{R}$ (respectively, $\left.\mathbb{S}_{p+1}^{n+2} \subset \mathbb{R}_{p+2}^{n+2} \times \mathbb{R}\right)$ 
defined by

$$
\varphi_{i}=\left(\sigma+\tau_{i} \nu, \tau_{i}\right)
$$

are marginally trapped.

Conversely any $n$-dimensional marginally trapped submanifold of $\mathbb{R}_{p+1}^{n+2}$ (respectively, $\mathbb{S}_{p+1}^{n+1}$ ) whose second fundamental form is not null is locally congruent to the image of such an immersion.

Remark 1. If the shape operator $A$ of $\sigma$ is diagonalizable (which is not always the case since the induced metric on $\sigma$ is not definite) the polynomial $P$ takes the form

$$
P(\tau):=\sum_{i=1}^{p} m_{i} \prod_{j \neq i}^{p}\left(\kappa_{j}^{-1}-\tau\right),
$$

where $\kappa_{1}, \ldots, \kappa_{p}, p \geq 2$ are the $p$ distinct, nonvanishing principal curvatures of $\sigma$ with multiplicity $m_{i}$.

In order to state the next theorem, we introduce some more notation: writing $x=\left(x^{\prime}, x^{\prime \prime}\right) \in \mathbb{R}^{n+2}=\mathbb{R}^{p+1} \times \mathbb{R}^{q+1}$, where $x^{\prime} \in \mathbb{R}^{p+1}$ and $x^{\prime \prime} \in \mathbb{R}^{q+1}$, we introduce the conjugation map $\overline{\left(x^{\prime}, x^{\prime \prime}\right)}:=\left(x^{\prime},-x^{\prime \prime}\right)$, as well as the $n \times n$ diagonal matrix $\overline{\operatorname{Id}}_{n}$ whose $(p, q)$-block decomposition is $\overline{\operatorname{Id}}_{n}=\left(\begin{array}{cc}\operatorname{Id}_{p} & 0 \\ 0 & -\mathrm{Id}_{q}\end{array}\right)$.

Since the normal spaces $N \Sigma$ are assumed to be two-dimensional and Lorentzian, the marginally trapped assumption $\langle\vec{H}, \vec{H}\rangle=0$ is equivalent to the fact that $\vec{H}$ is contained in one of the two null lines of $N \Sigma$. We shall call mean Gauss map, and denote by $v=\left(v^{\prime}, v^{\prime \prime}\right)$, the null vector which is collinear to $\vec{H}$ and normalized by the condition $v \in \mathbb{S}^{p} \times \mathbb{S}^{q} \subset \mathbb{R}^{p+1} \times \mathbb{R}^{q+1}$. The next two theorems give a global description of those marginally trapped submanifolds whose mean Gauss map has maximal rank. We observe that this is a generic property and that it is a stronger assumption than requiring the mean curvature vector $\vec{H}$ to have itself maximal rank. Theorem 3. Let $\Omega$ be an open subset of the universal covering of $\mathbb{S}^{p} \times \mathbb{S}^{q}$ and $\sigma \in C^{4}(\Omega)$. Denote by $\tau_{i}$ the roots of the polynomial of degree $n-1$

$$
P(\tau)=\operatorname{tr}\left(\left(\tau \operatorname{Id}_{n}+\sigma \overline{\operatorname{Id}}_{n}+2 \operatorname{Hess}(\sigma)\right)^{-1}\right) .
$$

Then the immersions

$$
\varphi_{i}: \Omega \rightarrow \mathbb{R}_{p+1}^{n+2}, \quad v \mapsto \tau_{i} v+\sigma \bar{v}+2 \nabla \sigma,
$$

are marginally trapped.

Conversely any connected, marginally trapped n-dimensional submanifold of $\mathbb{R}_{p+1}^{n+2}$ whose mean Gauss map $v$ has maximal rank is the image of such an immersion.

When $n=2$, the condition of maximal rank on $v$ is equivalent to the fact that the second fundamental form is not null. Hence Theorems 1 and 3 provide a complete characterization of marginally trapped surfaces of $\mathbb{R}^{4}$ with arbitrary signature. Since 
the Minkowski case has already been discussed in [Anciaux and Godoy 2012], we detail the case $(p, q)=(1,1)$, that is, of a Lorentzian surface in $\mathbb{R}_{2}^{4}$. Observe first that $\mathbb{R}_{2}^{4}$ is endowed with

(i) a natural pseudo-Kähler structure, with complex structure $J\left(x_{1}, x_{2}, x_{3}, x_{4}\right):=$ $\left(-x_{2}, x_{1},-x_{4}, x_{3}\right)$ and symplectic form $\omega=\langle J \cdot, \cdot\rangle=d x_{1} \wedge d x_{2}-d x_{3} \wedge d x_{4}$ this corresponds to the identification of $\mathbb{R}_{2}^{4}$ with $\mathbb{C}^{2}$ through the formula $\left(z_{1}, z_{2}\right)=\left(x_{1}+i x_{2}, x_{3}+i x_{4}\right)$;

(ii) a natural para-Kähler structure, with paracomplex ${ }^{1}$ structure

$$
K\left(x_{1}, x_{2}, x_{3}, x_{4}\right):=\left(x_{3}, x_{4}, x_{1}, x_{2}\right)
$$

and symplectic form $\omega^{\prime}=\langle K \cdot, \cdot\rangle=d x_{1} \wedge d x_{3}+d x_{2} \wedge d x_{4}$; this corresponds to the identification of $\mathbb{R}_{2}^{4}$ with $\mathbb{D}^{2}$, where $\mathbb{D}=\left\{a+e b \mid(a, b) \in \mathbb{R}^{2}\right\}$ is the ring of paracomplex numbers, through the formula $\left(w_{1}, w_{2}\right)=\left(x_{1}+e x_{3}, x_{2}+e x_{4}\right)$;

Corollary 1. Let $\Omega$ be an open subset of $\mathbb{R}^{2}$ endowed with the Lorentzian metric $d u^{2}-d v^{2}$ and $\sigma \in C^{4}(\Omega)$. Denote by subscript $u$ or $v$ the partial derivative with respect to the corresponding variable. Then the immersion

$\varphi: \Omega \rightarrow \mathbb{R}_{2}^{4} \simeq \mathbb{C}^{2}, \quad(u, v) \mapsto\left(\left(\sigma-\sigma_{u u}+\sigma_{v v}+2 i \sigma_{u}\right) e^{i u},\left(-\sigma-\sigma_{u u}+\sigma_{v v}-2 i \sigma_{v}\right) e^{i v}\right)$,

is weakly conformal and its null points are characterized by $\sigma+\sigma_{u u}+\sigma_{v v}= \pm 2 \sigma_{u v}$. Moreover, away from its null points, $\varphi$ is marginally trapped.

Conversely any connected, marginally trapped surface of $\mathbb{R}_{2}^{4}$ whose second fundamental form is not null is the image of such an immersion.

In [Chen 2009] and [Chen and Dillen 2007], marginally trapped surfaces of $\mathbb{R}_{2}^{4}$ which are in addition, respectively, flat and Lagrangian with respect to $\omega$ have been characterized. These additional conditions may be readily seen in terms of the formula given above:

Corollary 2. The marginally trapped immersion $\varphi$ of Corollary 1 is in addition

(i) flat if and only if $\left(\partial_{u u}-\partial_{v v}\right)\left(\left(\sigma+\sigma_{u u}+\sigma_{v v}\right)^{2}-4 \sigma_{u v}^{2}\right)=0$;

(ii) Lagrangian with respect to the symplectic form $\omega$ if and only if

$$
\sigma_{u}+\sigma_{v}+\sigma_{v v v}-\sigma_{u u v}-\sigma_{u v v}+\sigma_{u u u}=0 .
$$

Moreover there is no marginally trapped surface which is in addition Lagrangian with respect to the symplectic form $\omega^{\prime}$.

In the next theorem we give a characterization of marginally trapped submanifolds whose mean Gauss map has maximal rank in $\mathbb{S}_{p+1}^{n+2}$.

${ }^{1}$ We refer the reader to [Alekseevsky et al. 2009] or [Cruceanu et al. 1996] for material about paracomplex geometry, also called split geometry. 
Theorem 4. Let $\sigma: \mathcal{M} \rightarrow \mathbb{S}^{p+1} \times \mathbb{S}^{q}$ be an immersed, oriented hypersurface of class $C^{4}$ whose induced metric has signature $(p, q)$. Denote by $v$ its Gauss map (hence a $\mathbb{S}_{p+1}^{n+2}$-valued map) and by $A=-d v$ the corresponding shape operator. Denote by $\tau_{i}$ the roots of the polynomial of degree $n-1$

$$
P(\tau)=\operatorname{tr}(\tau \operatorname{Id}-A)^{-1} .
$$

Then the immersions $\varphi_{i}: \mathcal{M} \rightarrow \mathbb{S}_{p+1}^{n+2}$ defined by $\varphi_{i}:=v+\tau_{i} \sigma$ are marginally trapped.

Conversely any connected, marginally trapped $n$-dimensional submanifold of $\mathbb{S}_{p+1}^{n+2}$ whose mean Gauss map has maximal rank is the image of such an immersion.

Like in the flat case $\mathbb{R}_{2}^{4}$, a marginally trapped surface of $\mathbb{S}_{2}^{4}$ has either null second fundamental form, or a mean Gauss map with maximal rank. Therefore Theorems 1 and 4 provide a complete characterization in this case. It enjoys, moreover, a more explicit description:

Corollary 3. Let $\sigma$ be an immersion of class $C^{4}$ of a surface $M$ into $\mathbb{S}^{2} \times \mathbb{S}^{1}$ with Lorentzian induced metric. Denote by $v$ the Gauss map of $\sigma$ (hence a $\mathbb{S}_{2}^{4}$-valued map) and by $H$ the (scalar) mean curvature of $\sigma$ with respect to $v$. Then the immersion $\varphi: \mathcal{M} \rightarrow \mathbb{S}_{2}^{4}$ defined by

$$
\varphi=v+H \sigma
$$

is marginally trapped.

Conversely any connected marginally trapped surface of $\mathbb{S}_{2}^{4}$ whose second fundamental form is not null is the image of such an immersion.

\section{Submanifolds with null second fundamental form: proof of Theorem 1}

Let $\Sigma$ be an $n$-dimensional submanifold of $\mathbb{R}_{p+1}^{n+2}$ such that the induced metric on the normal bundle $N \Sigma$ is Lorentzian. Since the intersection of the light cone of $\mathbb{R}_{p+1}^{n+2}$ with $N \Sigma$ is made of two null lines, there exists a null normal frame, that is, a pair of normal, null vector fields along $\Sigma$ such that $\langle v, v\rangle=\langle\xi, \xi\rangle=0$ and $\langle v, \xi\rangle=2$. So, given a normal vector $N$, we have

$$
N=\frac{1}{2}(\langle N, \xi\rangle v+\langle N, v\rangle \xi)
$$

Lemma 1. The second fundamental form $h$ is collinear to $v$ (so in particular it is null) if and only if the mean curvature vector $\vec{H}$ is collinear to $v$ and $v$ has rank at most 1 .

Proof. We denote by $\left(e_{1}, \ldots, e_{n}\right)$ a local, orthonormal, tangent frame along $\Sigma$ and we set

$$
h_{i j}^{1}:=\left\langle h\left(e_{i}, e_{j}\right), v\right\rangle=-\left\langle d v\left(e_{i}\right), e_{j}\right\rangle
$$


Then we have, taking into account that $\langle d v, v\rangle=0$,

$$
d \nu\left(e_{i}\right)=-\sum_{i=1}^{n} h_{i j}^{1} e_{j}+\frac{1}{2}\left\langle d \nu\left(e_{i}\right), \xi\right\rangle \nu .
$$

Assume first that $h$ is collinear to $v$. Then clearly its trace $n \vec{H}$ is collinear to $v$ as well. Moreover all the coefficients $h_{i j}^{1}$ vanish, so by the equation above, for all $i$, $1 \leq i \leq n$, the vector $d v\left(e_{i}\right)$ is collinear to $v$, and hence $d v$ has rank at most 1 .

Conversely, if $d v$ has rank 1 , then $d v\left(e_{i}\right)$ and $d v\left(e_{j}\right)$ are proportional for any pair $(i, j), i \neq j$. Taking into account the symmetry of the tensor $h_{i j}^{1}$, an elementary calculation implies that there exist $n+1$ real constants $c, \lambda_{1}, \lambda_{2}, \ldots, \lambda_{n}$ such that $h_{i j}^{1}=c \lambda_{i} \lambda_{j}$. If in addition $\Sigma$ is marginally trapped, that is, $\langle n \vec{H}, v\rangle=$ $\operatorname{tr}\left[h_{i j}^{1}\right]_{1 \leq i, j \leq n}=c \sum_{i=1}^{n} \lambda_{i}^{2}=0$, then either $c=0$ or $\left(\lambda_{1}, \ldots, \lambda_{n}\right)=(0, \ldots, 0)$ and in both cases the whole tensor $h_{i j}^{1}$ vanishes, that is, $h$ is collinear to $v$.

We come back to the proof of Theorem 1, observing that under the assumption of the lemma above, $d v$ is collinear to $v$. This implies the existence of a map $\lambda \in C^{1}(\Sigma)$ such that $v=e^{\lambda} v_{0}$, where $v_{0}$ is a constant, null vector of $\mathbb{R}_{p+1}^{n+2}$ or $\mathbb{R}_{p+2}^{n+3}$. We conclude that $\Sigma \subset v_{0}^{\perp}$.

We now write the Gauss and the Ricci equations in the flat case:

$$
\begin{aligned}
\langle R(X, Y) Z, W\rangle+\langle h(X, Z), h(Y, W)\rangle-\langle h(X, W), h(Y, Z)\rangle & =0, \\
\left\langle R^{\perp}(X, Y) v, \xi\right\rangle-\left\langle\left[A_{v}, A_{\xi}\right] X, Y\right\rangle & =0,
\end{aligned}
$$

If $h$ is collinear to $v$, both terms $\langle h(X, Z), h(Y, W)\rangle$ and $\langle h(X, W), h(Y, Z)\rangle$ vanish, hence the curvature of the tangent bundle vanishes. Moreover, if $h$ is collinear to $v$, then $A_{v}$ vanishes as well and the normal bundle is flat.

In the case of $\mathbb{S}_{p+1}^{n+2}$, the Gauss and the Ricci equations become

$$
\begin{aligned}
\langle R(X, Y) Z, W\rangle+\langle h(X, Z), h(Y, W)\rangle-\langle h(X, W), h(Y, Z)\rangle & \\
& =\langle X, Z\rangle\langle Y, W\rangle-\langle X, W\rangle\langle Y, Z\rangle, \\
\left\langle R^{\perp}(X, Y) v, \xi\right\rangle-\left\langle\left[A_{\nu}, A_{\xi}\right] X, Y\right\rangle & =0 .
\end{aligned}
$$

Again, if $h$ is collinear to $v$, the terms $\langle h(X, Z), h(Y, W)\rangle$ and $\langle h(X, W), h(Y, Z)\rangle$ vanish. It follows that the scalar curvature of the induced metric is constant and equal to 1 . Analogously, the fact that $h$ is collinear to $v$ implies the vanishing of $A_{v}$ and therefore the flatness of the normal bundle.

\section{Parametrizations by hypersurfaces: proof of Theorem 2}

3.1. The flat case. Let $\varphi=(\psi, \tau)$ be an immersion of an $n$-dimensional manifold $M$ into $\mathbb{R}_{p+1}^{n+2}$ whose induced metric $\tilde{g}:=\varphi^{*}\langle\cdot, \cdot\rangle$ has signature $(p, q)$. In particular the induced metric on the normal space of $\bar{\varphi}$ is Lorentzian. Let $\tilde{v}$ be one of the two 
normalized, null normal fields along $\varphi$. Since the discussion is local, there is no loss of generality in assuming that, modulo congruence, its last component $v_{n+3}$ does not vanish, so that we may normalize $\tilde{v}=(v, 1)$.

Lemma 2. We set $\sigma:=\psi-\tau v$. Then the map $(\sigma, v): \mathcal{M} \rightarrow \mathbb{R}_{p+1}^{n+1} \times \mathbb{S}_{p}^{n}$ is an immersion.

Proof. Suppose $(\varphi, v)$ is not an immersion, so that there exists a nonvanishing vector $v \in T M$ such that $(d \varphi(v), d \nu(v))=(0,0)$. Since $d \psi=d \sigma+\tau d \nu+d \tau \nu$, it follows that

$$
d \varphi(v)=(d \psi(v), d \tau(v))=(d \tau(v) v, d \tau(v))=d \tau(v) \tilde{v},
$$

which is a normal to $\varphi$. However the immersion $\varphi$ is pseudo-Riemannian and therefore a vector cannot be tangent and normal at the same time, so we get the required contradiction.

\section{Lemma 3. $\quad\langle d \sigma, v\rangle=0$.}

Proof. Using again that $d \psi=d \sigma+\tau d \nu+d \tau \nu$ and observing that $\langle\nu, d \nu\rangle=0$, we have

$$
0=\langle d \varphi, \tilde{v}\rangle=\langle(d \psi, d \tau),(\nu, 1)\rangle=\langle d \psi, v\rangle-d \tau=\langle d \sigma, \nu\rangle .
$$

Lemma 4. Given $\epsilon>0$, there exists $t_{0} \in(-\epsilon, \epsilon)$ such that $\sigma+t_{0} v$ is an immersion, and $v$ is its Gauss map.

Proof. This follows from the fact that the set $\{t \in \mathbb{R} \mid \sigma+t v$ is not an immersion $\}$ contains at most $n$ elements. To see this, observe that given a pair of distinct real numbers $\left(t, t^{\prime}\right)$, we have

$$
\operatorname{Ker}(d \sigma+t d \nu) \cap \operatorname{Ker}\left(d \sigma+t^{\prime} d \nu\right)=\{0\}
$$

(otherwise we would have a contradiction with the fact that $(\sigma, v)$ is an immersion). Hence there cannot be more than $n$ distinct values $t$ such that $\operatorname{Ker}(d \sigma+t d v) \neq\{0\}$. The fact that $v$ is the Gauss map of $\sigma+t_{0} v$ comes from Lemma 3:

$$
\left\langle d\left(\sigma+t_{0} v\right), v\right\rangle=\langle d \sigma, v\rangle+t_{0}\langle d v, v\rangle=0 .
$$

Lemma 4 shows that there is no loss of generality in assuming that $\sigma$ is an immersion: if it is not the case, we may translate the immersion $\varphi$ along the vertical direction, setting $\varphi_{t_{0}}:=\varphi-\left(0, t_{0}\right)$. Of course $\varphi$ is marginally trapped if and only if $\varphi_{t_{0}}$ is so, and moreover the vector field $\tilde{v}$ is still normal to $\varphi_{t_{0}}$. Finally observe that the map $\sigma_{t_{0}}: \mathcal{M} \rightarrow \mathbb{R}_{p+1}^{n+1}$ associated to $\varphi_{t_{0}}$ is

$$
\sigma_{t_{0}}=\psi-\left(\tau-t_{0}\right) \nu=\psi-\tau \nu+t_{0} \nu=\sigma+t_{0} \nu,
$$

hence an immersion. 
We now describe the first fundamental form of $\varphi$ and its second fundamental form with respect to $\tilde{v}$, both in terms of the geometry of the immersion $\sigma$ :

Lemma 5. Denote by $g:=\sigma^{*}\langle\cdot, \cdot\rangle$ the metric induced on $M$ by $\sigma$ and $A$ the shape operator associated to $\mathrm{v}$.

Then the metric $\tilde{g}:=\varphi^{*}\langle\cdot, \cdot\rangle$ induced on $M$ by $\varphi$ is given by the formula

$$
\tilde{g}=g(\cdot, \cdot)-2 \tau g(A \cdot, \cdot)+\tau^{2} g(A \cdot, A \cdot) .
$$

In particular, the nondegeneracy assumption on $\tilde{g}$ implies that $\tau^{-1}$ is not equal to any principal curvature of $\varphi$. Moreover the second fundamental form of $\varphi$ with respect to $\tilde{v}$ is given by

$$
\tilde{h}_{\tilde{v}}:=\langle\tilde{h}(\cdot, \cdot), \tilde{v}\rangle=g(\cdot, A \cdot)-\tau g(A \cdot, A \cdot) .
$$

Proof. Since $\langle d \sigma, v\rangle=\langle d v, v\rangle=0$, given $v_{1}, v_{2} \in T M$, we have

$$
\begin{aligned}
\tilde{g}\left(v_{1}, v_{2}\right)= & \left\langle d \varphi\left(v_{1}\right), d \varphi\left(v_{2}\right)\right\rangle \\
= & \left\langle d \sigma\left(v_{1}\right), d \sigma\left(v_{2}\right)\right\rangle+\tau\left\langle d \sigma\left(v_{1}\right), d \nu\left(v_{2}\right)\right\rangle+\tau\left\langle d \nu\left(v_{1}\right), d \sigma\left(v_{2}\right)\right\rangle \\
& \quad+\tau^{2}\left\langle d v\left(v_{1}\right), d v\left(v_{2}\right)\right\rangle+d \tau\left(v_{1}\right) d \tau\left(v_{2}\right)\langle v, v\rangle-d \tau\left(v_{1}\right) d \tau\left(v_{2}\right) \\
= & g\left(v_{1}, v_{2}\right)-\tau\left(g\left(v_{1}, A v_{2}\right)+g\left(A v_{1}, v_{2}\right)\right)+\tau^{2} g\left(A v_{1}, A v_{2}\right) \\
= & g\left(v_{1}, v_{2}\right)-2 \tau g\left(A v_{1}, v_{2}\right)+\tau^{2} g\left(A v_{1}, A v_{2}\right) .
\end{aligned}
$$

We calculate the second fundamental form of $\varphi$ with respect to $\tilde{v}=(v, 1)$ :

$$
\begin{aligned}
\tilde{h}_{\tilde{v}} & =-\langle d \varphi, d \tilde{v}\rangle \\
& =-\langle d \sigma+\tau d \nu+d \tau \nu, d \nu\rangle=-\langle d \sigma, d \nu\rangle-\tau\langle d \nu, d v\rangle \\
& =g(\cdot, A \cdot)-\tau g(A \cdot, A \cdot) .
\end{aligned}
$$

The proof of Theorem 2 follows easily: denoting by $\tilde{A}_{\tilde{v}}$ the shape operator of $\varphi$ with respect to $\tilde{v}$, we have from Lemma 5

$$
g\left(\tilde{A}_{\tilde{\nu}}(\operatorname{Id}-\tau A) \cdot,(\operatorname{Id}-\tau A) \cdot\right)=g(\cdot,(\operatorname{Id}-\tau A) \cdot) .
$$

It follows that

$$
\tilde{A}_{\tilde{v}}:=(\operatorname{Id}-\tau A)^{-1}
$$

and that $\vec{H}$ is collinear to $\tilde{v}$ if and only if $\tilde{A}_{\tilde{v}}$ is trace-free, that is, $\tau$ is the root of the polynomial $P(\tau)=\operatorname{tr}(\operatorname{Id}-\tau A)^{-1}$.

Remark 2. If $\varphi$ is minimal, $\tau=0$ is a root of $P(\tau)$. The corresponding immersion $\varphi=(\sigma, 0)$ is not only marginally trapped but minimal. 
3.2. The $\mathbb{S}_{p+1}^{n+2}$ case. Let $\varphi=(\psi, \tau): \mathcal{M} \rightarrow \mathbb{S}_{p+1}^{n+2}$ an immersion such that the induced metric $\tilde{g}:=\varphi^{*}\langle\cdot, \cdot\rangle$ has signature $(p, q)$. Let $\tilde{v}$ be one of the two normalized, null normal fields along $\varphi$. Since the discussion is local, there is no loss of generality to assume that, modulo congruence, its last component $v_{n+3}$ does not vanish, so that we may normalize $\tilde{v}=(v, 1)$.

We define the null projection of $\varphi$ to be $\sigma:=\psi-\tau \nu$. The fact that $(\nu, 1) \in T_{\varphi} \mathbb{S}_{p+1}^{n+2}$, that is, $0=\langle(\psi, \tau),(v, 1)\rangle=\langle\psi, v\rangle-\tau$, implies that $\langle\psi, v\rangle=\tau$. Hence

$$
\begin{aligned}
\langle\sigma, \sigma\rangle & =\langle\psi, \psi\rangle-2 \tau\langle\psi, v\rangle+\tau^{2}\langle\nu, v\rangle \\
& =\langle\psi, \psi\rangle-\tau^{2} \\
& =\langle\varphi, \varphi\rangle \\
& =1
\end{aligned}
$$

which shows that $\sigma$ is $\mathbb{S}_{p}^{n+1}$-valued. The proofs of the next two lemmas are omitted, since they are similar to the flat case:

Lemma 6. The map $(\sigma, v): \mathcal{M} \rightarrow \mathbb{S}_{p+1}^{n+1} \times \mathbb{S}_{p+1}^{n+1}$ is an immersion.

$$
\text { Lemma 7. }\langle\sigma, v\rangle=0 \text { and }\langle d \sigma, v\rangle=0 .
$$

Unlike the flat case, there is no vertical translation in $\mathbb{S}_{p+1}^{n+2}$. We may however, up to an arbitrarily small perturbation, assume that $\sigma$ is an immersion.

Lemma 8. Given $\epsilon>0$, there exists $\alpha \in(-\epsilon, \epsilon)$ and a hyperbolic rotation $R^{\alpha}$ of angle $\alpha$ such that the null projection $\sigma^{\alpha}$ of $\varphi^{\alpha}:=R^{\alpha} \varphi$ is an immersion.

Proof. Set

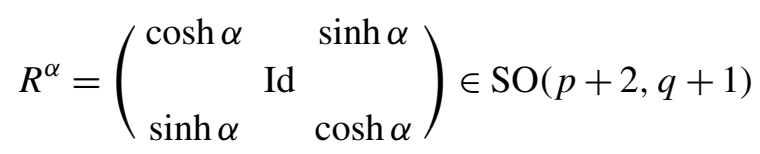

and $\varphi^{\alpha}:=R^{\alpha} \varphi, \tilde{v}^{\alpha}:=R^{\alpha} \tilde{v}$. Observe that $\tilde{v}^{\alpha}:=\left(v^{\alpha}, v_{n+3}^{\alpha}\right)$ is no longer normalized a priori, since its last component $v_{n+3}^{\alpha}$ is equal to $\cosh (\alpha)+\sinh (\alpha) v_{1}$, where $v_{1}$ is the first component of the vector $\tilde{v}$.

Nevertheless the null geodesic passing through the point $\varphi^{\alpha}$ and directed by the vector $\tilde{v}^{\alpha}$ crosses the slice $d \mathbb{S}_{p+2}^{n+2} \cap\left\{x_{n+3}=0\right\}$ at the point

$$
\left(\sigma^{\alpha}, 0\right):=\left(\psi^{\alpha}-\frac{\tau^{\alpha}}{v_{n+3}^{\alpha}} v^{\alpha}, 0\right) .
$$

Clearly $\sigma^{\alpha}$ is an immersion if and only if

$$
R^{-\alpha} \sigma^{\alpha}=\psi-\tau^{\alpha} \nu / \nu_{n+3}^{\alpha}=\varphi+\left(\tau-\tau^{\alpha} / \nu_{n+3}^{\alpha}\right) v
$$


is so. Observe that

$$
\begin{aligned}
\tau-\frac{\tau^{\alpha}}{v_{n+3}^{\alpha}} & =\tau-\frac{\cosh (\alpha) \tau+\sinh (\alpha) \psi_{1}}{\cosh (\alpha)+\sinh (\alpha) \nu_{1}} \\
& =\tanh (\alpha)\left(\psi_{1}-\tau v_{1}\right)+o(\alpha) \\
& =\sigma_{1} \alpha+o(\alpha)
\end{aligned}
$$

Now assume that $R^{-\alpha} \sigma^{\alpha}$ is not an immersion for $\alpha \in(-\epsilon, \epsilon)$. Hence there exists a one-parameter family of unit tangent vectors $v^{\alpha}$ such that

$$
0=d\left(R^{-\alpha} \sigma^{\alpha}\right)\left(v^{\alpha}\right)=d \sigma\left(v^{\alpha}\right)+\left(d \sigma_{1}\left(v^{\alpha}\right) v+\sigma_{1} d v\left(v^{\alpha}\right)\right) \alpha+o(\alpha)
$$

for all $\alpha \in(-\epsilon, \epsilon)$. Thus

$$
\left\{\begin{array}{l}
d \sigma\left(v^{\alpha}\right)=0 \\
d \sigma_{1}\left(v^{\alpha}\right) v+\sigma_{1} d v\left(v^{\alpha}\right)=\sigma_{1} d v\left(v^{\alpha}\right)=0 .
\end{array}\right.
$$

By Lemma $6, d v\left(v^{\alpha}\right)$ and $d \sigma\left(v^{\alpha}\right)$ cannot vanish simultaneously, therefore $\sigma_{1}$ vanishes. Repeating the argument with suitable rotations yields that all the other coordinates of $\sigma$ vanish, a contradiction since $\sigma \in \mathbb{S}_{p+1}^{n+1}$.

By the previous lemma we may assume that $\sigma$ is an immersion. The remainder of the proof follows the lines of that of the flat case; in particular, Lemma 5 still holds.

\section{Parametrization by the mean Gauss map}

4.1. The flat case: proof of Theorem 3. In this section $\Sigma$ denotes an $n$-dimensional submanifold of $\mathbb{R}_{p+1}^{n+2}$ whose induced metric has signature $(p, q)$ and such that the normalized vector $v \in \mathbb{S}^{p} \times \mathbb{S}^{q} \subset \mathbb{R}^{n+2}$ has rank $n$. We may therefore parametrize $\Sigma$ locally by $\nu$, that is, by a map $\varphi: \Omega \rightarrow \mathbb{R}_{p+1}^{n+2}$, where $\Omega$ is an open subset of the universal covering of $\mathbb{S}^{p} \times \mathbb{S}^{q}$. We set $\sigma(v):=\frac{1}{2}\langle\varphi(v), v\rangle$ and $\tau(v):=\frac{1}{2}\langle\varphi(v), \bar{v}\rangle .^{2}$

Lemma 9. We have

$$
\varphi=\tau \nu+\sigma \bar{v}+2 \nabla \sigma
$$

where $\nabla$ is the gradient with respect to the induced metric on $\mathbb{S}^{p} \times \mathbb{S}^{q}$ (that is, $\nabla \sigma=\left(\nabla^{\prime} \sigma,-\nabla^{\prime \prime} \sigma\right)$, where $\nabla^{\prime}$ and $\nabla^{\prime \prime}$ are respectively the gradients on $\mathbb{S}^{p}$ and $\left.\mathbb{S}^{q}\right)$.

Proof. Since $v$ and $\bar{v}$ are null and $\langle v, \bar{v}\rangle=2$, we clearly have $\varphi=\tau v+\sigma \bar{v}+V$, where $V \in T_{\nu}\left(\mathbb{S}^{p} \times \mathbb{S}^{q}\right)=T_{\bar{\nu}}\left(\mathbb{S}^{p} \times \mathbb{S}^{q}\right)=T_{\nu^{\prime}} \mathbb{S}^{p} \times T_{\nu^{\prime \prime}} \mathbb{S}^{q}$. In order to determine $V$, we use the assumption $\langle d \varphi, v\rangle=0$. Taking into account that

$$
d \varphi=d \tau \nu+\tau d \nu+d \sigma \bar{v}+\sigma d \bar{\nu}+d V
$$

\footnotetext{
${ }^{2}$ The pair $(\sigma, \tau)$ may be regarded as a generalization of the support function of a hypersurface.
} 
and that $\langle\nu, v\rangle,\langle d v, v\rangle$ and $\langle d \bar{v}, v\rangle$ vanish, we have

$$
\langle d \varphi, v\rangle=d \sigma\langle\bar{v}, v\rangle+\langle d V, \nu\rangle=2 d \sigma+\langle d V, \nu\rangle .
$$

On the other hand, from $0=d(\langle V, v\rangle)=\langle d V, v\rangle+\langle V, d v\rangle$, we conclude, observing that $d v=\mathrm{Id}$,

$$
\langle V, W\rangle=\langle V, d v(W)\rangle=-\langle d V(W), v\rangle=2 d \sigma(W) \text { for all } W \in T_{\nu}\left(\mathbb{S}^{p} \times \mathbb{S}^{q}\right),
$$

which, by the very definition of the gradient, proves that $V=2 \nabla \sigma$.

We now complete the proof of Theorem 3: define the endomorphism $A_{\nu}$ on $T_{v}\left(\mathbb{S}^{p} \times \mathbb{S}^{q}\right)$ by

$$
\left\langle A_{v} \cdot, \cdot\right\rangle=h_{v} .
$$

Hence, using that $d v$ is the identity map of $T_{v}\left(\mathbb{S}^{p} \times \mathbb{S}^{q}\right)$, we have

$$
\left\langle d \varphi \circ A_{\nu} \cdot d \varphi \cdot\right\rangle=-\langle d \nu \cdot, d \varphi \cdot\rangle=-\langle\Pi \cdot, d \varphi \cdot\rangle,
$$

where $\Pi$ is the restriction to $T_{\nu}\left(\mathbb{S}^{p} \times \mathbb{S}^{q}\right)$ of the normal projection $\mathbb{R}_{p+1}^{n+2} \rightarrow T_{\varphi(\nu)} \mathscr{Y}$. It follows that

$$
d \varphi \circ A_{v}=-\Pi
$$

and therefore

$$
A_{v}^{-1}=-\Pi^{-1} \circ d \varphi
$$

(the maximal rank assumption on $v$ implies that $\Pi$ is one-to-one). In order to calculate the trace of $A_{v}$ we introduce an orthonormal basis $\left(e_{1}, \ldots, e_{n}\right)$ of $T_{v}\left(\mathbb{S}^{p} \times \mathbb{S}^{q}\right)$, such that $\left\langle e_{i}, e_{i}\right\rangle=1$ if $1 \leq i \leq p$ and $\left\langle e_{i}, e_{i}\right\rangle=-1$ if $p+1 \leq i \leq n$. We define the coefficients $a_{i j}$ by

$$
d \varphi\left(e_{i}\right)=\sum_{j=1}^{n} a_{i j} \Pi e_{j} .
$$

Clearly

$$
A_{v}^{-1}=\left[a_{i j}\right]_{1 \leq i, j \leq n} .
$$

To determine the coefficients $a_{i j}$ explicitly, we calculate

$$
d \varphi=d \tau \nu+\tau d \nu+d \sigma \bar{\nu}+\sigma d \bar{\nu}+2 d \nabla \sigma .
$$

Then we introduce a null, normal vector field $\xi$ along $\mathscr{Y}$ such that $(\nu, \xi)$ is a null frame of $N \Sigma=T \Sigma^{\perp}$, which is in addition normalized, that is, $\langle\nu, \xi\rangle=2$. Then the projection of a vector $V$ of $\mathbb{R}_{p+1}^{n+2}$ onto $N \Sigma$ is given by the formula

$$
\frac{1}{2}(\langle V, \xi\rangle v+\langle V, v\rangle \xi) .
$$

It follows that

$$
\Pi V=V-\frac{1}{2}(\langle V, \xi\rangle \nu+\langle V, v\rangle \xi)
$$


For $1 \leq i \leq p$, using (1) and observing that $d v\left(e_{i}\right)=d \bar{v}\left(e_{i}\right)=e_{i}$, we have

$$
d \varphi\left(e_{i}\right)=(\tau+\sigma) e_{i}+d \tau\left(e_{i}\right) \nu+d \sigma\left(e_{i}\right) \bar{\nu}+2 d(\nabla \sigma)\left(e_{i}\right)
$$

Using (2) and the fact that $\left\langle d(\nabla \sigma)\left(e_{i}\right), e_{j}\right\rangle=\operatorname{Hess}(\sigma)\left(e_{i}, e_{j}\right)$, we conclude that, for $1 \leq i \leq p$,

$$
a_{i j}=\delta_{i j}(\tau+\sigma)+2 \operatorname{Hess}(\sigma)\left(e_{i}, e_{j}\right) .
$$

Analogously we get, if $p+1 \leq i \leq n$,

$$
a_{i j}=\delta_{i j}(\tau-\sigma)+2 \operatorname{Hess}(\sigma)\left(e_{i}, e_{j}\right) .
$$

The conclusion of the proof of Theorem 3 follows easily.

4.2. The case $(p, q)=(1,1)$ : proof of Corollaries 1 and 2 . We use the natural identification $\mathbb{R}_{2}^{4} \simeq \mathbb{C}^{2}$ and denote by $(u, v)$ the natural coordinates on $\mathbb{S}^{1} \times \mathbb{S}^{1}$, so that $v:=\left(e^{i u}, e^{i v}\right)$. In particular, the metric on $\mathbb{S}^{1} \times \mathbb{S}^{1}$ is $d u^{2}-d v^{2}$. Hence

$$
A_{v}=-\left(\begin{array}{cc}
\tau+\sigma+2 \sigma_{u u} & 2 \sigma_{u v} \\
-2 \sigma_{u v} & \tau-\sigma-2 \sigma_{v v}
\end{array}\right)^{-1}
$$

whose trace is $2 / \operatorname{det} A_{v}\left(\tau+\sigma_{u u}-\sigma_{v v}\right)$. Hence $\varphi$ is marginally trapped if and only if $\tau=\sigma_{v v}-\sigma_{u u}$.

We now study the induced metric $\varphi^{*}\langle\cdot, \cdot\rangle$. Since

$$
\varphi=\tau\left(e^{i u}, e^{i v}\right)+\sigma\left(e^{i u},-e^{i v}\right)+2\left(i \sigma_{u} e^{i u},-i \sigma_{v} e^{i v}\right),
$$

we have

$$
\begin{aligned}
& \varphi_{u}=\left(\left((\tau-\sigma)_{u}+i\left(2 \sigma_{u u}+\tau+\sigma\right)\right) e^{i u},\left((\tau-\sigma)_{u}-2 i \sigma_{u v}\right) e^{i v}\right), \\
& \varphi_{v}=\left(\left((\tau+\sigma)_{v}+2 i \sigma_{u v}\right) e^{i u},\left((\tau+\sigma)_{v}+i\left(-2 \sigma_{v v}+\tau-\sigma\right)\right) e^{i v}\right) .
\end{aligned}
$$

By a straightforward calculation the coefficients of the first fundamental form $\varphi^{*}\langle\cdot, \cdot\rangle$ are

$$
\begin{aligned}
& E:=\left(2 \sigma_{u u}+\tau+\sigma\right)^{2}-4 \sigma_{u v}^{2}, \\
& F:=4 \sigma_{u v}\left(\sigma_{u u}-\sigma_{v v}+2 \tau\right), \\
& G:=-\left(2 \sigma_{v v}-\tau+\sigma\right)^{2}+4 \sigma_{u v}^{2} .
\end{aligned}
$$

The marginally trapped assumption $\tau=\sigma_{v v}-\sigma_{u u}$ implies

$$
E=-G=\left(2 \sigma_{u u}+\tau+\sigma\right)^{2}-4 \sigma_{u v}^{2}=\left(\sigma+\sigma_{u u}+\sigma_{v v}\right)^{2}-4 \sigma_{u v}^{2}
$$

and the vanishing of $F$, so that $\varphi$ is weakly conformal (and conformal whenever $E$ does not vanish).

It is well known that the induced metric of a surface with isothermic coordinates is flat if and only if its conformal factor is harmonic. Here we are dealing with the 
Lorentzian metric $d u^{2}-d v^{2}$, whose Laplacian operator is $\partial_{u u}-\partial_{v v}$. Hence the induced metric is flat if and only if

$$
\left(\partial_{u u}-\partial_{v v}\right) E=\left(\partial_{u u}-\partial_{v v}\right)\left(\left(\sigma+\sigma_{u u}+\sigma_{v v}\right)^{2}-4 \sigma_{u v}^{2}\right) .
$$

Marginally trapped Lagrangian surfaces. We recall that $J\left(z_{1}, z_{2}\right)=\left(i z_{1}, i z_{2}\right)$, so

$$
J \varphi_{u}=\left(-\left(2 \sigma_{u u}+\tau+\sigma+i(\tau-\sigma)_{u}\right) e^{i u},\left(2 \sigma_{u v}+i(\tau-\sigma)_{u}\right) e^{i v}\right) .
$$

Hence, using the usual formula $\omega=\langle J \cdot, \cdot\rangle$,

$$
\begin{aligned}
\omega\left(\varphi_{u}, \varphi_{v}\right)= & \left\langle J \varphi_{u}, \varphi_{v}\right\rangle \\
= & -\left(2 \sigma_{u u}+\tau+\sigma\right)(\tau+\sigma)_{v}+2 \sigma_{u v}(\tau-f)_{u}-2 \sigma_{u v}(\tau+\sigma)_{v} \\
& \quad-(\tau-\sigma)_{u}\left(-2 \sigma_{v v}+\tau-\sigma\right) \\
= & -(\tau+\sigma)_{v}\left(\sigma+\sigma_{u u}+\sigma_{v v}+2 \sigma_{u v}\right)+(\tau-\sigma)_{u}\left(\sigma+\sigma_{u u}+\sigma_{v v}+2 \sigma_{u v}\right) \\
= & \left(\sigma+\sigma_{u u}+\sigma_{v v}+2 \sigma_{u v}\right)\left(-\sigma_{v}-\sigma_{u}-\sigma_{v v v}+\sigma_{u u v}+\sigma_{u v v}-\sigma_{u u u}\right) .
\end{aligned}
$$

The first factor does not vanish except at degenerate points, so $\varphi$ is Lagrangian with respect to $\omega$ if and only if $\sigma_{v}+\sigma_{u}+\sigma_{v v v}-\sigma_{u u v}-\sigma_{u v v}+\sigma_{u u u}=0$.

Recalling that the paracomplex structure is given by

$$
K\left(x_{1}, x_{2}, x_{3}, x_{4}\right):=\left(x_{3}, x_{4}, x_{1}, x_{2}\right),
$$

we have

$$
K \varphi_{u}=\left(\left((\tau-\sigma)_{u}-2 i \sigma_{u v}\right) e^{i v},\left((\tau-\sigma)_{u}+i\left(2 \sigma_{u u}+\tau+\sigma\right)\right) e^{i u}\right),
$$

and so

$$
\begin{aligned}
\omega^{\prime}\left(\varphi_{u}, \varphi_{v}\right) & =\left\langle K \varphi_{u}, \varphi_{v}\right\rangle \\
& =\cos (u-v)\left(-4 \sigma_{u v}^{2}-\left(\tau-\sigma-2 \sigma_{v v}\right)\left(2 \sigma_{u u}+\tau+\sigma\right)\right) \\
& =\cos (u-v)\left(\left(-4 \sigma_{u v}^{2}+\left(\sigma+\sigma_{u u}+\sigma_{v v}\right)\right)^{2}\right. \\
& =\cos (u-v) E .
\end{aligned}
$$

Hence $\varphi$ is Lagrangian with respect to $\omega^{\prime}$ if and only if the induced metric is totally null, which is incompatible with the marginally trapped assumption.

4.3. The case of $\mathbb{S}_{p+1}^{n+2}$ : proof of Theorem 4. Let $\varphi: \mathcal{M} \rightarrow \mathbb{S}_{p+1}^{n+2}$ be an immersed submanifold of codimension two of $\mathbb{S}_{p+1}^{n+2}$. Let $\sigma$ be a normal, null vector field along $\varphi$ which is normalized in such way that $\sigma \in \mathbb{S}^{p+1} \times \mathbb{S}^{q}$. We moreover assume that $\sigma$ has maximal rank, that is, $\sigma: \mathcal{M} \rightarrow \mathbb{S}^{p+1} \times \mathbb{S}^{q}$ is an immersed hypersurface.

Lemma 10 [Godoy and Salvai 2013]. There exists a unique pair $(\nu, \tau)$, where $v: \mathcal{M} \rightarrow \mathbb{S}_{p+1}^{n+2}$ and $\tau \in C^{2}(\mathcal{M})$ are such that

$$
\varphi=\nu+\tau \sigma .
$$


Moreover the map $v: \mathcal{M} \rightarrow \mathbb{S}_{p+1}^{n+2}$ is the Gauss map of $\sigma$; that is, $v \in T_{\sigma}\left(\mathbb{S}^{p+1} \times \mathbb{S}^{q}\right)$ and $\langle d \sigma, v\rangle=0$.

Proof. For an arbitrary $\tau \in C^{2}(\mathcal{M})$, we have $v:=\varphi-\tau \sigma \in \mathbb{S}_{p+1}^{n+2}$. Hence we shall determine $\tau \in C^{2}(M)$ by the condition $v \in T_{\nu}\left(\mathbb{S}^{p+1} \times \mathbb{S}^{q}\right)$. Recalling the decomposition $\mathbb{R}^{n+3}=\mathbb{R}^{p+2} \times \mathbb{R}^{q+1}$, and writing $v=\left(v^{\prime}, v^{\prime \prime}\right), \sigma=\left(\sigma^{\prime}, \sigma^{\prime \prime}\right)$ accordingly, this condition amounts to $\left\langle v^{\prime}, \sigma^{\prime}\right\rangle_{p+2}=0$ and $\left\langle v^{\prime \prime}, \sigma^{\prime \prime}\right\rangle_{q+1}=0$, where $\langle\cdot, \cdot\rangle_{p+2}$ and $\langle\cdot, \cdot\rangle_{q+1}$ denote the Euclidean inner products of $\mathbb{R}^{p+2}$ and $\mathbb{R}^{q+1}$, respectively. These two equations yield $\tau=\left\langle v^{\prime}, \sigma^{\prime}\right\rangle_{p+1}$ and $\tau=\left\langle v^{\prime \prime}, \sigma^{\prime \prime}\right\rangle_{q+1}$, which are actually two equivalent requirements since $\langle\varphi, \sigma\rangle=\left\langle\varphi^{\prime}, \sigma^{\prime}\right\rangle_{p+2}-\left\langle\varphi^{\prime \prime}, \sigma^{\prime \prime}\right\rangle_{q+1}$ vanishes. Therefore $\tau$ is uniquely determined by the condition $v \in T_{\sigma}\left(\mathbb{S}^{p+1} \times \mathbb{S}^{q}\right)=T_{\sigma^{\prime}} \mathbb{S}^{p+1} \times \mathbb{T}_{\sigma^{\prime \prime}} \mathbb{S}^{q}$.

It remains to check that $v$ is the Gauss map of $\sigma$. For this purpose we differentiate $\varphi=v+\tau \sigma$ and remember that $\sigma$ is normal to $\varphi$, so that

$$
0=\langle d \varphi, \sigma\rangle=\langle d \nu, \sigma\rangle+d \tau\langle\sigma, \sigma\rangle+\tau\langle d \sigma, \sigma\rangle=\langle d \nu, \sigma\rangle .
$$

Hence $\langle d \nu, \sigma\rangle$ vanishes. Since $0=d(\langle\nu, \sigma\rangle)=\langle d \nu, \sigma\rangle+\langle\nu, d \sigma\rangle$, we deduce that $\langle d \sigma, v\rangle$ vanishes as well.

Observe that the lemma above implies furthermore that the induced metric $g:=\sigma^{*}\langle\cdot, \cdot\rangle$ is nondegenerate, since $\sigma(\mathcal{M})$ is a hypersurface and admits a unit normal vector field.

Lemma 11. Denote by $g:=\sigma^{*}\langle\cdot, \cdot\rangle$ the metric induced on $M$ by $\sigma$, and by $A$ the shape operator associated to $v$, so $A(v):=-d v(v)$ for all $v \in T M$. Then the metric $\tilde{g}:=\varphi^{*}\langle\cdot, \cdot\rangle$ induced on $M$ by $\varphi$ is given by the formula

$$
\tilde{g}=\tau^{2} g(\cdot, \cdot)-2 \tau g(A \cdot, \cdot)+g(A \cdot, A \cdot) .
$$

In particular, the nondegeneracy assumption on $\tilde{g}$ implies that $\tau$ is not equal to any principal curvature of $v$. Moreover the second fundamental form of $\varphi$ with respect to $\sigma$ is given by

$$
h_{\sigma}:=\langle h(\cdot, \cdot), \sigma\rangle=g(A \cdot, \cdot)-\tau g(\cdot, \cdot) .
$$

Proof. Taking into account that $d \varphi=d \nu+d \tau \sigma+\tau d \sigma$, we have

$$
\begin{aligned}
\tilde{g} & =\langle d \varphi, d \varphi\rangle=\langle d \nu, d \nu\rangle+2 \tau\langle d \nu, d \sigma\rangle+\tau^{2}\langle d \sigma, d \sigma\rangle \\
& =g(A \cdot, A \cdot)-2 \tau g(A \cdot, \cdot)+\tau^{2} g(\cdot, \cdot)
\end{aligned}
$$

and

$$
h_{\sigma}=-\langle d \varphi, d \sigma\rangle=-\langle d \nu, d \sigma\rangle-\tau\langle d \sigma, d \sigma\rangle=g(A \cdot, \cdot)-\tau g(\cdot, \cdot) .
$$

The proof of Theorem 4 is now straightforward: if $\varphi$ is marginally trapped, we may assume without loss of generality that its mean curvature vector $\vec{H}$ is collinear to $\sigma$. By the maximal rank assumption on $\sigma$ we may use Lemmas 10 and 11 . 
Denote by $\tilde{A}_{\sigma}$ the shape operator of $\varphi$ with respect to $\sigma$. Then, from Lemma 11 above, we have

$$
g\left(\tilde{A}_{\sigma}(\tau \mathrm{Id}-A) \cdot,(\tau \mathrm{Id}-A) \cdot\right)=g(\cdot,(\tau \mathrm{Id}-A) \cdot) .
$$

It follows that $\tilde{A}_{\sigma}:=(\tau \mathrm{Id}-A)^{-1}$ and that $\vec{H}$ is collinear to $v$ if and only if $A_{v}$ is trace-free, that is, $\tau$ is the root of the polynomial $P(\tau)=\operatorname{tr}(\tau \mathrm{Id}-A)^{-1}$.

4.4. The case $(p, q)=(1,1)$ : proof of Corollary 3. It is straightforward that if $M$ is a $2 \times 2$ matrix, then $\operatorname{tr} M^{-1}=(\operatorname{det} M)^{-1} \operatorname{tr} M$. Hence $\operatorname{tr}(\tau \operatorname{Id}-A)^{-1}$ vanishes if and only if $\operatorname{tr}(\tau \operatorname{Id}-A)=2 \tau-\operatorname{tr} A$ does. Hence $\varphi$ is marginally trapped if and only if $\tau=\operatorname{tr} A / 2:=H$, the (scalar) mean curvature of the immersion $\sigma$. This proves Corollary 3 .

\section{Further remarks}

5.1. Interpretation of the result in terms of contact geometry. The constructions in the previous sections come from the natural contact structure enjoyed by the spaces of null geodesics of the ambient spaces and from the fact that the set of null geodesics which are normal to a submanifold of codimension two is Legendrian with respect to this contact structure.

The proof of Theorem 2 is based on the following fact: Let $U$ be the dense, open subset of null geodesics of $\mathbb{R}_{p+1}^{n+2}$ that cross the horizontal hyperplane $\left\{x_{n+2}=0\right\}$ (in the Minkowski case $(p, q)=(n, 0)$, all null geodesics cross the horizontal hyperplane). Then the correspondence $\{(\sigma, 0)+t(\nu, 1) \mid t \in \mathbb{R}\} \mapsto(\sigma, \nu)$ defines a contactomorphism between $U$ and the unit tangent bundle $T^{1} \mathbb{R}_{p}^{n+1}$. The canonical contact structure $\alpha$ of the unit tangent of a pseudo-Riemannian manifold $(\mathcal{M}, g)$ is given by the expression $\alpha=g(d \sigma, v)$, where $v$ is a unit vector tangent to $\mathcal{M}$ at the point $\sigma$. Hence, given an immersion $x \mapsto(\sigma(x), v(x))$ of an $n$-dimensional manifold such that $x \mapsto \sigma(x)$ is an immersion as well (a generic assumption), the Legendre condition $g\left(d \sigma_{x}, v(x)\right)=0$ simply means that $\nu$ is the Gauss map of $\sigma$ or, equivalently, $v$ is a unit vector field normal to the immersed hypersurface $\sigma$.

The interpretation of the proof of Theorem 3 in terms of contact geometry is as follows: The space of null geodesics of $\mathbb{R}_{p+1}^{n+2}$ may be identified with space of one-jets on $\mathbb{S}^{p} \times \mathbb{S}^{q}$, that is, the space $T\left(\mathbb{S}^{p} \times \mathbb{S}^{q}\right) \times \mathbb{R}$ such that to the triple $(\nu, V, z) \in T\left(\mathbb{S}^{p} \times \mathbb{S}^{q}\right) \times \mathbb{R}$, we associate the null line $\{V+z \bar{v}+t v \mid t \in \mathbb{R}\} \subset \mathbb{R}_{p+1}^{n+2}$. The natural contact structure on the space of one-jets $T M \times \mathbb{R}$, where $(M, g)$ is a pseudo-Riemannian manifold, is given by $\alpha:=\psi-d z$, where $\psi$ is the Liouville form $^{3}$ or tautological form on TM. Moreover a generic Legendrian immersion

\footnotetext{
${ }^{3}$ To be more precise, the Liouville form is canonically defined on the cotangent bundle $T^{*} \mu$ of a differentiable manifold $\mathcal{M}$. If $\mathcal{M}$ is moreover equipped with a pseudo-Riemannian metric (as it is the case of $\mathbb{S}^{p} \times \mathbb{S}^{q}$ ), we may identify $T^{*} \mathcal{M}$ and $T M$ and therefore speak of a Liouville form on $T M$.
} 
in $T M \times \mathbb{R}$ is locally a section and takes the form $v \mapsto(v, \nabla \sigma(v), \sigma(v))$, where $\sigma \in C^{2}(M)$ and $\nabla$ denotes the gradient of the metric $g$. It follows, in the case $M=\mathbb{S}^{p} \times \mathbb{S}^{q}$, that a generic Legendrian congruence of null lines of $\mathbb{R}_{p+1}^{n+2}$ takes the form

$$
v \mapsto\{\nabla \sigma(v)+\sigma(v) \bar{v}+t v \mid t \in \mathbb{R}\},
$$

where $\sigma \in C^{2}\left(\mathbb{S}^{p} \times \mathbb{S}^{q}\right)$. The choice of real function $\tau \in C^{2}\left(\mathbb{S}^{p} \times \mathbb{S}^{q}\right)$ determines an $n$-dimensional submanifold parametrized by $v \mapsto \nabla \sigma(v)+\sigma(v) \bar{v}+\tau(v) v$, one of whose null normals is $v$. These observations inspired the proof of Theorem 3 .

Finally the proof of Theorem 4 comes from the fact, proved in [Godoy and Salvai 2013], that the space of null geodesics of $\mathbb{S}_{p+1}^{n+2}$ can be identified with $T^{1}\left(\mathbb{S}^{p} \times \mathbb{S}^{q}\right)$, the unit tangent bundle of $\mathbb{S}^{p} \times \mathbb{S}^{q}$, as follows: to the pair $(\nu, \psi) \in T^{1}\left(\mathbb{S}^{p} \times \mathbb{S}^{q}\right)$, we associate the null line $\{\psi+t v \mid t \in \mathbb{R}\} \subset \mathbb{S}_{p+1}^{n+2}$.

5.2. Relation between Theorems 2 and 3 in the case $(p, q)=(n, 0)$. In the Lorentzian case $(p, q)=(n, 0)$, it is easy to relate the formulas of Theorems 2 and 3. To avoid confusion, all mathematical quantities from Theorem 2 will be written with subscript 2 , and those from Theorem 3 with subscript 3 . We start by writing $v_{3}=\left(v_{2}, 1\right) \in \mathbb{S}^{n} \times \mathbb{S}^{0} \simeq \mathbb{S}^{n} \times\{1,-1\}$, so that $\bar{v}_{3}=\left(v_{2},-1\right)$. Hence the main formula of Theorem 3 becomes

$$
\varphi=\left(\left(\tau_{3}+\sigma_{3}\right) \nu_{2}+2 \nabla \sigma_{3}, \tau_{3}-\sigma_{3}\right),
$$

where $\sigma_{3} \in C^{4}\left(\mathbb{S}^{n} \times \mathbb{S}^{0}\right) \simeq C^{4}\left(\mathbb{S}^{n}\right)$ and $\tau_{3}$ depends on the second derivatives of $\sigma_{3}$. Introducing $\sigma_{2}:=2 \sigma_{3} \nu_{2}+2 \nabla \sigma_{3}$ and $\tau_{2}:=\tau_{3}-\sigma_{3}$, we obtain

$$
\varphi=\left(\sigma_{2}+\tau_{2} \nu_{2}, \tau_{2}\right),
$$

which is exactly the main formula of Theorem 2. Observe that $\left\langle d \sigma_{2}, v_{2}\right\rangle$ vanishes, that is, $v_{2}$ is normal to the immersion $\sigma_{2}$, which is therefore parametrized by its Gauss map. Moreover $\left\langle\sigma_{2}, v_{2}\right\rangle=2 \sigma_{3}$, that is, $2 \sigma_{3}$ is the support function of the immersion $\sigma_{2}$.

\section{References}

[Alekseevsky et al. 2009] D. V. Alekseevsky, K. Medori, and A. Tomassini, “Однородные паракэлеровы многообразия Эйнштейна”, Uspekhi Mat. Nauk 64:1(385) (2009), 3-50. Translated as "Homogeneous para-Kähler Einstein manifolds" in Russian Math. Surveys 64:1 (2009), 1-43. MR 2010k:53068 Zbl 1179.53050

[Anciaux and Godoy 2012] H. Anciaux and Y. Godoy, "Marginally trapped submanifolds in Lorentzian space forms and in the Lorentzian product of a space form by the real line", preprint, 2012. arXiv 1209.5118

[Chen 2009] B.-Y. Chen, "Classification of marginally trapped surfaces of constant curvature in Lorentzian complex plane”, Hokkaido Math. J. 38:2 (2009), 361-408. MR 2010f:53092 Zbl 1187. 53056 
[Chen and Dillen 2007] B.-Y. Chen and F. Dillen, "Classification of marginally trapped Lagrangian surfaces in Lorentzian complex space forms", J. Math. Phys. 48:1 (2007), Art. ID \#013509. MR 2008d:53097 Zbl 1121.53047

[Chruściel et al. 2010] P. T. Chruściel, G. J. Galloway, and D. Pollack, "Mathematical general relativity: a sampler”, Bull. Amer. Math. Soc. (N.S.) 47:4 (2010), 567-638. MR 2011j:53152 Zbl 1205.83002

[Cruceanu et al. 1996] V. Cruceanu, P. Fortuny, and P. M. Gadea, "A survey on paracomplex geometry”, Rocky Mountain J. Math. 26:1 (1996), 83-115. MR 97c:53112 Zbl 0856.53049

[Georgiou and Guilfoyle 2014] N. Georgiou and B. Guilfoyle, "Marginally trapped surfaces in spaces of oriented geodesics", J. Geom. Phys. 82 (2014), 1-12. MR 3206636 Zbl 06300570 arXiv 1305.6600

[Godoy and Salvai 2013] Y. Godoy and M. Salvai, "The canonical contact structure on the space of oriented null geodesics of pseudospheres and products", Adv. Geom. 13:4 (2013), 713-722. MR 3181543 Zbl 06229240

[Kriele 1999] M. Kriele, Spacetime: foundations of general relativity and differential geometry, Lecture Notes in Physics 59, Springer, Berlin, 1999. MR 2001g:53126 Zbl 0933.83001

[Penrose 1965] R. Penrose, "Gravitational collapse and space-time singularities", Phys. Rev. Lett. 14 (1965), 57-59. MR 30 \#2897 Zbl 0125.21206

Received September 23, 2013. Revised February 20, 2014.

Henri AnCIAUX

InSTITUto DE MATEMÁtica e Estatística

UNIVERSIDADE DE SÃo PAULO

RUA DO MATÃO, 1010

CIDADE UNIVERSITÁRIA

05508-090 São PaUlo, SP

BRAZIL

henri@ime.usp.br 


\title{
PACIFIC JOURNAL OF MATHEMATICS
}

\author{
msp.org/pjm
}

Founded in 1951 by E. F. Beckenbach (1906-1982) and F. Wolf (1904-1989)

\section{EDITORS}

Don Blasius (Managing Editor)

Department of Mathematics

University of California

Los Angeles, CA 90095-1555

blasius@math.ucla.edu

\author{
Paul Balmer \\ Department of Mathematics \\ University of California \\ Los Angeles, CA 90095-1555 \\ balmer@math.ucla.edu \\ Robert Finn \\ Department of Mathematics \\ Stanford University \\ Stanford, CA 94305-2125 \\ finn@math.stanford.edu \\ Sorin Popa \\ Department of Mathematics \\ University of California \\ Los Angeles, CA 90095-1555 \\ popa@math.ucla.edu
}

\author{
Vyjayanthi Chari \\ Department of Mathematics \\ University of California \\ Riverside, CA 92521-0135 \\ chari@math.ucr.edu \\ Kefeng Liu \\ Department of Mathematics \\ University of California \\ Los Angeles, CA 90095-1555 \\ liu@math.ucla.edu \\ Jie Qing \\ Department of Mathematics \\ University of California \\ Santa Cruz, CA 95064 \\ qing@ cats.ucsc.edu
}

\section{PRODUCTION}

Silvio Levy, Scientific Editor, production@msp.org

\section{SUPPORTING INSTITUTIONS}

ACADEMIA SINICA, TAIPEI

CALIFORNIA INST. OF TECHNOLOGY

INST. DE MATEMÁTICA PURA E APLICADA

KEIO UNIVERSITY

MATH. SCIENCES RESEARCH INSTITUTE

NEW MEXICO STATE UNIV.

OREGON STATE UNIV.

\author{
STANFORD UNIVERSITY \\ UNIV. OF BRITISH COLUMBIA \\ UNIV. OF CALIFORNIA, BERKELEY \\ UNIV. OF CALIFORNIA, DAVIS \\ UNIV. OF CALIFORNIA, LOS ANGELES \\ UNIV. OF CALIFORNIA, RIVERSIDE \\ UNIV. OF CALIFORNIA, SAN DIEGO \\ UNIV. OF CALIF., SANTA BARBARA
}

\author{
Daryl Cooper \\ Department of Mathematics \\ University of California \\ Santa Barbara, CA 93106-3080 \\ cooper@math.ucsb.edu \\ Jiang-Hua Lu \\ Department of Mathematics \\ The University of Hong Kong \\ Pokfulam Rd., Hong Kong \\ jhlu@maths.hku.hk \\ Paul Yang \\ Department of Mathematics \\ Princeton University \\ Princeton NJ 08544-1000 \\ yang@math.princeton.edu
}

These supporting institutions contribute to the cost of publication of this Journal, but they are not owners or publishers and have no responsibility for its contents or policies.

See inside back cover or msp.org/pjm for submission instructions.

The subscription price for 2014 is US $\$ 410 /$ year for the electronic version, and \$535/year for print and electronic.

Subscriptions, requests for back issues and changes of subscribers address should be sent to Pacific Journal of Mathematics, P.O. Box 4163, Berkeley, CA 94704-0163, U.S.A. The Pacific Journal of Mathematics is indexed by Mathematical Reviews, Zentralblatt MATH, PASCAL CNRS Index, Referativnyi Zhurnal, Current Mathematical Publications and Web of Knowledge (Science Citation Index).

The Pacific Journal of Mathematics (ISSN 0030-8730) at the University of California, c/o Department of Mathematics, 798 Evans Hall \#3840, Berkeley, CA 94720-3840, is published twelve times a year. Periodical rate postage paid at Berkeley, CA 94704, and additional mailing offices. POSTMASTER: send address changes to Pacific Journal of Mathematics, P.O. Box 4163, Berkeley, CA 94704-0163.

PJM peer review and production are managed by EditFLOW ${ }^{\circledR}$ from Mathematical Sciences Publishers.

\section{PUBLISHED BY}

\section{mathematical sciences publishers \\ nonprofit scientific publishing}

http://msp.org/

(C) 2014 Mathematical Sciences Publishers 


\section{PACIFIC JOURNAL OF MATHEMATICS}

Volume $272 \quad$ No. $2 \quad$ December 2014

Marginally trapped submanifolds in space forms with arbitrary

signature

HENRI ANCIAUX

One line complex Kleinian groups

275

Waldemar Barrera, Angel Cano and JuAn Pablo

NAVARRETE

A note on flux integrals over smooth regular domains

305

IDO BRIGHT and JOHN M. LEE

On stable commutator length in hyperelliptic mapping class groups

323

DANny Calegari, NaOyuKi Monden and Masatoshi Sato

Compositions of birational endomorphisms of the affine plane

PierRetTe CASSOU-NoguÈs and DANIEl DAigle

The Bochner formula for isometric immersions

395

ALESSANDRO SAVO

On solutions to Cournot-Nash equilibria equations on the sphere

423

MICAH WARREN

Double and triple Givental's J-functions for stable quotients invariants

439

ALEKSEY ZiNGER

Acknowledgement 